\title{
Nachweis und Abgrenzung von Pseudoallergien auf Arzneimittel
}

Allergische und pseudoallergische Reaktionen sind als weitgehend dosisunabhängige und nicht stoffspezifische Effekte von unerwünschten Arzneimittelwirkungen abzugrenzen. Die nicht immunologisch vermittelten pseudoallergischen Reaktionen können klinisch fast jedes allergische Symptom imitieren. Anders als allergische Phänomene können sie jedoch bereits beim Erstkontakt mit dem betreffenden Stoff auftreten.

$\mathrm{H}$ äufig manifestieren sich Pseudoallergien als generalisierte Urtikaria und Quincke-Ödem oder als anaphylaktoide Reaktion unterschiedlichen Schweregrades bis hin zum anaphylaktischen Schock. Auch Exantheme werden oft beobachtet. Grundsätzlich können pseudoallergische Reaktionen alle vier Typen immunologischer Reaktionen nach Coombs und Gell imitieren, erklärte D. Vieluf, Hamburg. Die zugrunde liegenden Mechanismen sind bei den meisten Reaktionen noch nicht aufgedeckt; als Pathomechanismen diskutiert werden unter anderem direkte Mediatorfreisetzung oder Komplementaktivierung, neuro-psychogene Reflexe sowie embolisch-toxische Reaktionen.

\section{Diagnostik auf vier Säulen}

Die Abklärung unklarer Reaktionen auf Arzneimittel basiert auf den vier Säulen der allergischen Diagnostik: Anamnese, Hauttest, In-vitroDiagnostik und Provokationstestung.

Im Rahmen der Anamnese ist nach bekannten Allergien sowie früheren Unverträglichkeitsreaktionen $\mathrm{zu}$ fragen. Besonders genau muß man sich über Zubereitung und Anwendungsform des applizierten Arzneimittels informieren, um sämtliche Inhaltsstoffe lückenlos, also inklusive Hilfsstof- fen, zu ermitteln. Auch aus der Medikationsdauer läßt sich ein Anhaltspunkt gewinnen: Neu verordnete Medikamente sind häufiger Auslöser einer Pseudoallergie als solche, die bereits längere Zeit eingenommen werden. Schließlich sollte zum Ausschluß einer Intoxikation die genaue Dosierung ermittelt werden.

Nach Erhebung der ausführlichen Krankengeschichte erfolgt auch bei vermuteter pseudoallergischer Reaktion eine Hauttestung (in der Reihenfolge Epikutan-, Prick-, Scratch-, unter Umständen auch Intrakutantest).

„Zu vermuten ist, daß einige der als ,pseudoallergisch“ eingestuften Reaktionen in Wirklichkeit allergischer Natur sind und wir die Allergie nur deshalb nicht als solche erkennen, weil die im Organismus entstehenden Metaboliten nicht bekannt sind, gegen welche die Sensibilisierung gerichtet ist." (D. Vieluf)

Zur In-vitro-Diagnostik von Pseudoallergien stehen noch keine etablierten Verfahren zur Verfügung: Der Basophilen-Histamin-Freisetzungs-Test ist eher wenig aussagekräftig. Strittig ist noch, inwieweit der CAST-ELISA, also eine Kombination des zellulären Antigen-StimulationsTests mit dem ELISA, sowohl IgEvermittelte als auch nicht IgE-vermittelte Reaktionen nachweisen kann.
Die Provokation als letzte mögliche diagnostische Maßnahme erfolgt meist subkutan oder oral. Erforderlich wird sie beispielsweise dann, wenn der Patient nicht ein, sondern - wie dies häufig der Fall ist - mehrere Medikamente eingenommen hatte.

Mögliche psychophysiologische Reaktionen können durch plazebokontrollierte Provokationstests ausgeschlossen werden. Umgekehrt ist bei negativem Ausgang in Betracht zu ziehen, daß möglicherweise die Expositionsdauer gegenüber dem Medikament zu kurz war.

Kontraindikationen gegen eine Provokation sind Schwangerschaft oder nicht sicher medikamentös beherrschbare Erkrankungen wie ein Status asthmaticus.

\section{Auch Ausweichpräparate testen!}

Weisen Patienten z.B. eine Unverträglichkeit gegen ASS auf, muß sorgfältig geprüft werden, welches andere Präparat für den Patienten alternativ in Frage kommt, erklärte Vieluf: In Hamburg gaben 10\% der 720 untersuchten Patienten mit ASS-Intoleranz an, auch Paracetamol nicht zu vertragen, und in der Provokationstestung reagierten knapp 16\% der Patienten mit ASS-Pseudoallergie anaphylaktoid auf Paracetamol.

Cave: Vor dem Hauttest immer nach den gegenwärtig eingenommenen Medikamenten fragen! Besonders wichtig ist es, darüber Bescheid zu wissen, ob der Patient Betablocker einnimmt. Diese Medikation ist eine absolute Kontraindikation gegen Hauttests: Zum einen können diese Substanzen anaphylaktoide Reaktionen verstärken, zum anderen ihre Behandlung beeinträchtigen.

(wpa)

Quelle: Symposium „Arzneimittel-Allergien“ (unterstützt durch die Firma DPC-Biermann, Bad Nauheim) im Rahmen der 20. Tagung der Deutschen Gesellschaft für Allergie- und Immunitätsforschung, Freiburg, 2. bis 5. Oktober 1996. 